\title{
Non-communicating Mucinous Biliary Cystadenoma as a Rare Cause of Jaundice
}

\author{
Alicia M Alvarez, Kanwarpreet Singh Tandon, Andrew Ukleja, Pablo Bejarano and Luis F Lara* \\ Department of Gastroenterology, Digestive Diseases Institute, Cleveland Clinic Florida, USA
}

\begin{abstract}
Biliary cystadenomas with ovarian like mesenchymal stroma are rare benign cystic neoplasms of liver which have premalignant potential. A 58-year-old woman presented for the evaluation of a $3.6 \mathrm{~cm}$ cyst compressing the hepatic duct, jaundice and abnormal liver enzymes. Endoscopic retrograde cholangiopancreatography (ERCP) and stent placement provided symptomatic improvement. The patient was successfully treated by laparoscopic common bile duct excision with cholecystectomy choledochoduodenostomy.
\end{abstract}

Keywords: Biliary cystic neoplasm; Cystadenoma; Mucinous cystadenoma; Ovarian like stroma

\section{Introduction}

Biliary cystic tumors are rare hepatic and extrahepatic biliary neoplasms with the potential for malignant transformation. Mucinous biliary cystadenoma (MBC) account for less than $5 \%$ of the reported cystic lesions originating from the bile ducts, and mainly affect women over age 40 [1-3]. Presentation is non-specific including abdominal pain and fullness, nausea, increased abdominal girth, and rarely a palpable mass [14]. Cross sectional imaging may reveal the cystadenoma and occasionally the internal septations or papillary infoldings of the cyst itself.

$\mathrm{MBC}$ are usually multilocular with an epithelial lining composed of biliary type cuboidal or nonciliated columnar glandular epithelium surrounded by an intermediary subepithelial layer of mesenchymal cells resembling ovarian stroma ("ovarian like" stroma) in the cyst wall which is a differentiating characteristic, and usually do not communicate with the bile duct lumen. The pathogenesis is unknown but may be congenital from aberrant biliary duct or misplaced germ cells.

A case of a patient with jaundice and pruritus diagnosed with an extra-hepatic mucinous biliary cystadenoma is presented with a review of the specialty literature.

\section{Case Report}

A 58 year old healthy female presented with jaundice and pruritus, as well as elevated liver enzymes, and dilated bile duct on abdominal ultrasound. Magnetic resonance imaging (MRI) showed intra hepatic duct dilation and a cystic dilation of the common hepatic duct measuring $2.4 \mathrm{~cm} \times 2.5 \mathrm{~cm}$. Endoscopic retrograde cholangiopancreatography (ERCP) revealed a stricture at the common hepatic duct near the bifurcation, and cholangioscopy and directed biopsies were unremarkable. Placement of a biliary stent resulted in a significant clinical improvement. Endoscopic ultrasound showed a $3.6 \mathrm{~cm}$ exophytic cyst compressing the common hepatic duct. $10 \mathrm{cc}$ of mucinous fluid was aspirated. Amylase was $<2 \mathrm{U} / \mathrm{L}$ and Carcinoembryonic antigen (CEA) $1.3 \mathrm{mg} / \mathrm{dL}$. The pancreas was normal.

Choledochal cyst, duplication cyst and cholangiocarcinoma were considered in the differential, and the lack of symptoms and stability of the lesion favored a benign diagnosis. Repeat blood tests were unremarkable. MRI showed a decreased size of the cystic lesion at the porta hepatis. ERCP was repeated to remove/replace the biliary stent. Cholangiogram showed luminal narrowing at the left hepatic duct take off with a fixed, round opacification occluding $75 \%$ of the lumen, and proximal to this was a second stricture where the lumen narrowed up to 95\%. Brushings were negative. A biliary stent was successfully placed. Due to the persistence of biliary obstruction, a laparoscopic common bile duct excision with cholecystectomy and choledochoduodenostomy was performed. A mass adherent to the anterior wall of common bile duct was removed.

On gross pathology the mass measured $3.3 \mathrm{~cm} \times 2.4 \mathrm{~cm} \times 1.6 \mathrm{~cm}$. It was a cystic structure filled with fluid and described to have a smooth lining. A section through this cystic structure showed additional cysts that were apparent within the wall of the mass, with the largest measuring 0.4 $\mathrm{cm}$ in greatest dimension. On histology, the lining of the cysts were flat to cuboidal epithelial cells that lack dysplastic features and mitotic activity. The underlying stroma was composed of slender spindle cells reminiscent of ovarian-like stroma admixed with small blood vessels and collagen. The stromal cells lacked atypia and they were positive for estrogen receptors by immunohistochemistry (Figures 1 and 2).

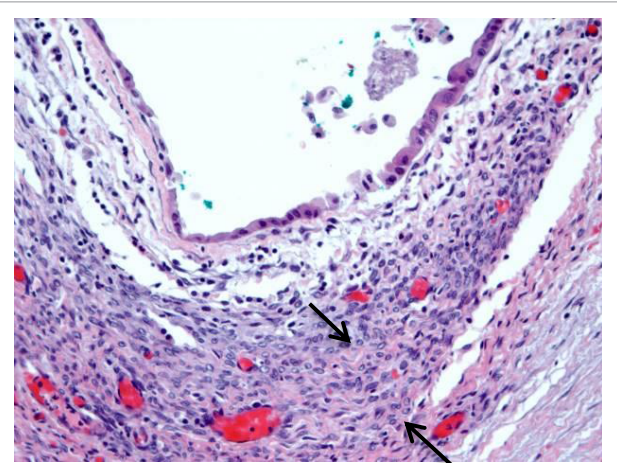

Figure 1: Mucinous cystadenoma showing a cuboidal epithelium lining and underlying stroma of elongated cells. (Hematoxylin and eosin; 100X). Spindle stromal cells between arrows.

*Corresponding author: Luis F Lara, Department of Gastroenterology, Cleveland Clinic Florida, 2950, Cleveland Clinic Blvd, Weston, Florida 33331, USA, Tel: (954) 659-5646; Fax: (954)659-5647; E-mail: LARAL@ccf.org

Received August 26, 2015; Accepted September 15, 2015; Published September 17, 2015.

Citation: Alvarez AM, Tandon KS, Ukleja A, Bejarano P, Lara LF (2015) Noncommunicating Mucinous Biliary Cystadenoma as a Rare Cause of Jaundice. J Cytol Histol 6: 369. doi:10.4172/2157-7099.1000369

Copyright: (c) 2015 Alvarez AM, et al. This is an open-access article distributed under the terms of the Creative Commons Attribution License, which permits unrestricted use, distribution, and reproduction in any medium, provided the original author and source are credited. 


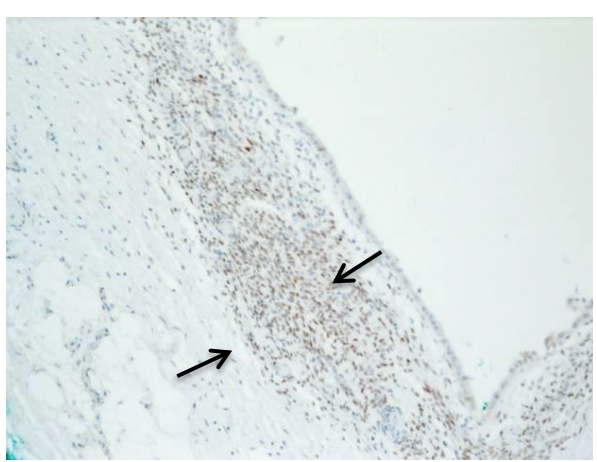

Figure 2: Immunohistochemistry for estrogen receptors showing nuclear positive staining in the stromal cells.(Peroxidase; 100X)

\section{Discussion}

Cystadenomas of the biliary tract are rare neoplasia affecting middle age females $[3,5]$. There are two types of biliary cystadenomas: mucinous and serous. The mucinous type is more common and is divided into subtypes on the basis of the presence or absence of mesenchymal stroma [2]. Absence of mesenchymal stroma increases the malignant potential, especially in men, and may indicate another subtype of mucinous cystadenoma [1].

The mucin producing biliary cystadenomas can be divided into the columnar or cuboidal types. The columnar type shows a pattern of villous papillae resembling intestine. The cuboidal type has a pancreaticobiliary and/or oncocytic pattern. Macroscopically they are divided into the cystic type or the ductectatic type, which presents with a diffusely dilated bile duct [6]. They are likely congenital, presumably a proliferation of ectopic embryonic tissues that forms bile ducts and the gallbladder [7]. It is still not clear if communication with the bile duct is of clinical importance or may even represent a different disease as is seen with mucin producing cysts of the pancreas, where pancreas mucinous neoplasms and intra-papillary mucinous neoplasms of the pancreas are distinct pathologies with a different prognosis and treatment approach [8].

There are no specific signs and symptoms of this disease, and diagnosis is usually fortuitous, but patients may present with abdominal pain, nausea, fullness, increased abdominal girth and palpable mass. Jaundice, pruritus and elevation of liver enzymes are rare.

Commonly used methods for diagnosis include ultrasound and computerized tomography (CT), which show focal lesions sometimes with internal septae, but magnetic cholangioresonance provides better images of the lesion and thus the reference test of choice. ERCP is usually insufficient to establish a diagnosis but may be useful to treat biliary obstruction and jaundice as was our case.

The differential diagnosis is broad and includes cholangiocarcinoma, complicated hepatic cyst, duplication cyst, biliary cystadenocarcinoma, mesenchymal hamartoma and hydatid cyst [9]. Surgically appropriate patients should undergo an excision to confirm the diagnosis, and resection is usually curative. Aspiration, marsupialization, internal drainage, partial excision and enucleation are associated with almost universal recurrence and occasional malignant degeneration [1,10-12].

The malignant potential is not known but likely exists extrapolating data from mucinous adenomas from other parts of the body. Mucinous biliary cystadenoma should be expected when radiologic imaging studies suggest a multilocular cystic intra or extra-hepatic lesion close to or arising from the bile duct, especially in a woman.

\section{Conclusion}

The diagnosis of $\mathrm{MBC}$ requires a high degree of suspicion and a multi-disciplinary approach to consider the diagnosis. Surgical removal of the lesion along with margins is the current treatment of choice to confirm the diagnosis and for curative purposes, especially when causing biliary obstruction. They are probably premalignant also and thus should be considered for removal.

\section{References}

1. Devaney K, Goodman ZD, Ishak KG (1994) Hepatobiliary cystadenoma and cystadenocarcinoma. A light microscopic and immunohistochemical study of 70 patients. Am J Surg Pathol 18: 1078-1091.

2. Wheeler DA, Edmondson HA (1985) Cystadenoma with mesenchymal stroma (CMS) in the liver and bile ducts. A clinicopathologic study of 17 cases, 4 with malignant change. Cancer 56: 1434-1445.

3. Ishak KG, Willis GW, Cummins SD, Bullock AA (1977) Biliary cystadenoma and cystadenocarcinoma: report of 14 cases and review of the literature. Cancer 39: 322-338.

4. Buetow PC, Buck JL, Pantongrag-Brown L, Ros PR, Devaney K, et al. (1995) Biliary cystadenoma and cystadenocarcinoma: clinical-imaging-pathologic correlations with emphasis on the importance of ovarian stroma. Radiology 196: 805-810.

5. Davies W, Chow M, Nagorney D (1995) Extrahepatic biliary cystadenomas and cystadenocarcinoma. Report of seven cases and review of the literature. Ann Surg 222: 619-625.

6. Shibahara H, Tamada S, Goto M, Oda K, Nagino M, et al. (2004) Pathologic features of mucin-producing bile duct tumors: two histopathologic categories as counterparts of pancreatic intraductal papillary-mucinous neoplasms. The American journal of surgical pathology 28: 327-338.

7. Subramony C, Herrera GA, Turbat-Herrera EA (1993) Hepatobiliary cystadenoma. A study of five cases with reference to histogenesis. Arch Pathol Lab Med 117: 1036-1042.

8. Zen Y, Fujii T, Itatsu K, Nakamura K, Konishi F, et al. (2006) Biliary cystic tumors with bile duct communication: a cystic variant of intraductal papillary neoplasm of the bile duct. Mod Pathol 19: 1243-1254.

9. Choi BI, Lim JH, Han MC, Lee DH, Kim SH, et al. (1989) Biliary cystadenoma and cystadenocarcinoma: CT and sonographic findings. Radiology 171: 57-61.

10. Preetha M, Chung AY, Lim-Tan SK, Lim DT, Thng CH (2004) Intrahepatic biliary cystadenoma presenting with obstructive jaundice. Asian J Surg 27: 243-245.

11. Lau WY, Chow CH, Leung ML (1990) Total excision of mucinous biliary cystadenoma. Aust N Z J Surg 60: 226-228.

12. Thomas KT, Welch D, Trueblood A, Sulur P, Wise P, et al. (2005) Effective treatment of biliary cystadenoma. Ann Surg 241: 769-773. 